\title{
Recent results at the $\phi$-meson region from the CMD-3 detector at the VEPP-2000 collider
}

\author{
Vyacheslav Ivanov ${ }^{*}$, Evgeny Solodov ${ }^{1}$, Evgeny Kozyrev $^{1}$, and Georgiy Razuvaev ${ }^{1}$ \\ ${ }^{1}$ Budker Institute of Nuclear Physics, 11, akademika Lavrentieva prospect, Novosibirsk, 630090 \\ Russia
}

\begin{abstract}
This report is devoted to the recent results of the study of the processes $e^{+} e^{-} \rightarrow K_{S} K_{L}, K^{+} K^{-}, \pi^{+} \pi^{-} 2 \pi^{0}, 2 \pi^{+} 2 \pi^{-}, \eta \gamma$ at the $\phi$-meson region with the CMD-3 detector at the VEPP-2000 collider. From the combined analysis of $K_{S} K_{L}$ and $K^{+} K^{-}$final states the $\phi$-meson parameters were measured, and the obtained ratio of $g_{\phi K^{+} K^{-}}$and $g_{\phi K_{S} K_{L}}$ couplings was found to be compatible with the isospin symmetry. From the analyzes of the $\pi^{+} \pi^{-} 2 \pi^{0}$ and $2 \pi^{+} 2 \pi^{-}$final states the branching ratios $\mathcal{B}(\phi \rightarrow$ $\left.\pi^{+} \pi^{-} 2 \pi^{0}\right)$ and $\mathcal{B}\left(\phi \rightarrow 2 \pi^{+} 2 \pi^{-}\right)$were determined.
\end{abstract}

\section{Introduction}

A high precision measurement of the inclusive $e^{+} e^{-} \rightarrow$ hadrons cross section is required for a calculation of the hadronic contribution to the muon anomalous magnetic moment $(g-2)_{\mu}$ in the frame of the Standard Model. To confirm or deny the observed difference between the calculated [1] and measured [2] $(g-2)_{\mu}$ values more precise measurements of the exclusive channels of $e^{+} e^{-} \rightarrow$ hadrons are necessary. On the other hand, the study of the exclusive final states can provide useful information about the precision of the isospin symmetry, particle parameters, branching ratios and coupling constants.

\section{VEPP-2000 collider and CMD-3 detector}

The VEPP-2000 $e^{+} e^{-}$collider [3] at the Budker Institute of Nuclear Physics (Novosibirsk, Russia) covers the center-of-mass energy $\left(E_{c . m .}\right)$ range from 0.32 to $2.0 \mathrm{GeV}$ and employs a novel technique of round beams to reach luminosity up to $10^{32} \mathrm{~cm}^{-2} \mathrm{~s}^{-1}$ at $2.0 \mathrm{GeV}$. The Cryogenic Magnetic Detector (CMD-3), described in [4], is installed in one of the two beams interaction regions. The detector tracking system consists of the cylindrical drift chamber (DC) and double-layer cylindrical multiwire proportional Z-chamber, installed inside a thin superconducting solenoid with 1.0-1.3 T magnetic field. Both subsystems are also used to provide the trigger signals. DC contains 1218 hexagonal cells in 18 layers and allows one to measure charged particle momentum with $1.5-4.5 \%$ accuracy in the $40-1000$ $\mathrm{MeV} / \mathrm{c}$ range, and the polar $\theta$ and azimuthal $\varphi$ angles with $20 \mathrm{mrad}$ and 3.5-8.0 mrad accuracy, respectively. Amplitude information from the DC wires is used to measure the

*Corresponding author: vyacheslav_lvovich_ivanov@mail.ru 
ionization losses $d E / d x$ of charged particles. The barrel electromagnetic calorimeters based on liquid xenon ( $\mathrm{LXe})\left(5.4 \mathrm{X}_{0}\right)$ and CsI crystals $\left(8.1 \mathrm{X}_{0}\right)$ are placed outside the solenoid [5]. The total amount of material in front of the barrel calorimeter is $0.13 \mathrm{X}_{0}$ that includes the solenoid as well as the radiation shield and vacuum vessel walls. BGO crystals of $13.4 \mathrm{X}_{0}$ thicknesses are used in the endcap calorimeter. The magnetic flux return yoke is surrounded by scintillation counters which are used to tag cosmic events.

The energy range $E_{c . m}=920-1070 \mathrm{MeV}$ was scanned in 2013 year, and $9.8 \mathrm{pb}^{-1}$ of integral luminosity in 22 energy points was collected. The integral luminosity at each $E_{c . m}$. point was determined using the events of the processes $e^{+} e^{-} \rightarrow e^{+} e^{-}$and $e^{+} e^{-} \rightarrow 2 \gamma$ with about $1 \%$ accuracy [6]. The beam energy was monitored by using the Back-ScatteringLaser-Light system [7].

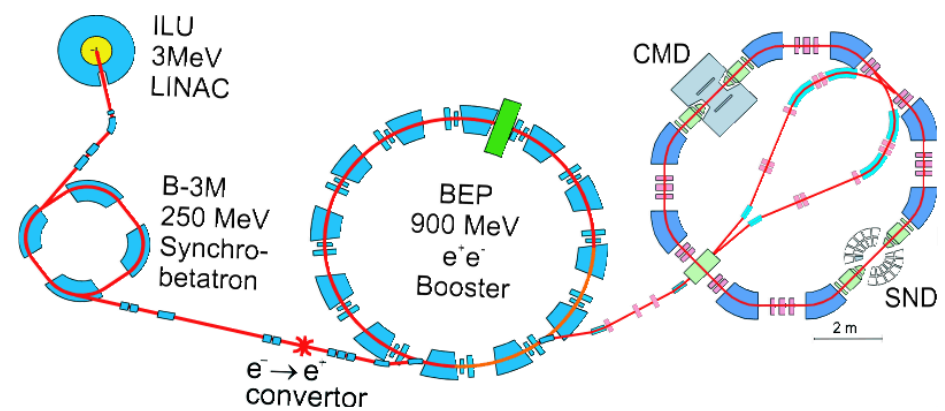

Fig. 1. VEPP-2000 collider layout.
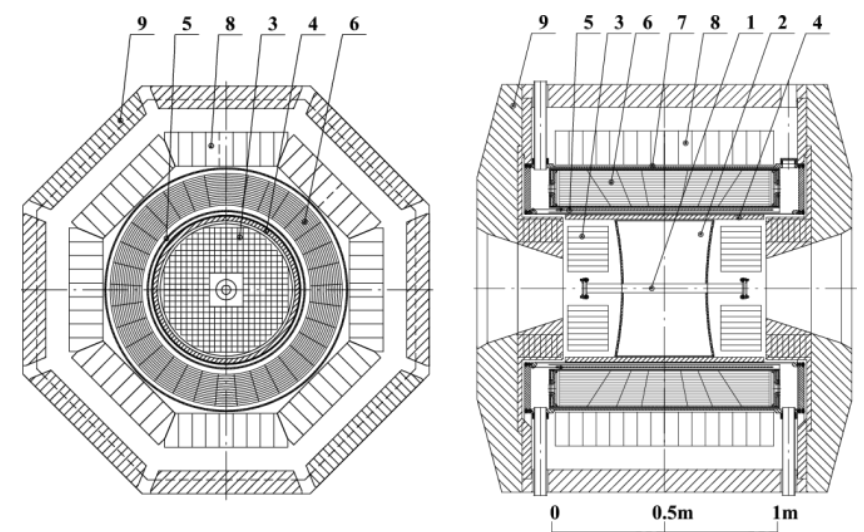

Fig. 2. CMD-3 detector layout. 1 - beam pipe, 2 - drift chamber, 3 - BGO endcap calorimeter, 4 Z-chamber, 5 - superconducting solenoid, 6 - LXe calorimeter, 7 - time-of-flight system, 8 - CsI calorimeter, 9 - yoke.

The main goals of the VEPP-2000 complex are: to achieve $<1 \%$ systematic error of the cross section measurement for the major exclusive $e^{+} e^{-} \rightarrow$ hadrons channels; to study other exclusive processes, especially those which were not measured before and were taken into account in the calculation of $(g-2)_{\mu}$ via the isotopic relations; to measure the parameters of "excited" vector mesons like $\rho^{\prime}, \rho^{\prime \prime}, \omega^{\prime}, \phi^{\prime}$ etc.; to test the conservation of vector current; to study the nucleon electromagnetic form factors; to study some of the diphoton processes (e.g. $e^{+} e^{-} \rightarrow \eta^{\prime}$ ).

It should be noted that the study of $\phi$-meson energy range is not a priority of CMD-3, but $\phi$ is very important for studying of photon, pion and kaon detection efficiencies and for studying the $\pi^{ \pm}$energy depositions in the calorimeters (necessary for proper $\pi / \mu$ 
separation). On the other hand, CMD-3 can provide interesting results on some problems, where the systematic uncertainty is decisive.

\section{Study of the $e^{+} e^{-} \rightarrow K_{S} K_{L}, K^{+} K^{-}$processes at $\phi$}

To select the charged kaons, we demand the event to have 2 tracks in the DC, passing from the beams interaction region and satisfying the collinearity conditions ||$\varphi_{1}-\varphi_{2}|-\pi|<$ $0.3 \mathrm{rad}$ and $\left|\theta_{1}+\theta_{2}-\pi\right|<0.3 \mathrm{rad}$. Then the almost background-free sample of signal $K^{+} K^{-}$events with charged kaons are extracted using $d E / d x$ losses (see Fig. 3).

The signal identification for the $K_{S} K_{L}$ final state is based on the detection of two pions from the $K_{S} \rightarrow \pi^{+} \pi^{-}$decay. The longitudinal distance $z_{v}$ and the transverse coordinate $\rho_{v}$ of the vertex should be less than 10 and $6 \mathrm{~cm}$ correspondingly. Pions from $K_{S}$ decay are required to have polar angles $1 \mathrm{rad}<\theta_{\pi}<(\pi-1) \mathrm{rad}$. Then, the $d E / d x$ vs. particle momenta distribution is used to select the signal events (see Fig. 4). The signal/background separation is performed by the fitting of the distribution of invariant mass of two pions $m_{\text {inv }}\left(\pi^{+}, \pi^{-}\right)$(see Fig. 5). The major background process was found to be $e^{+} e^{-} \rightarrow$ $\pi^{+} \pi^{-} 2 \pi^{0}$.

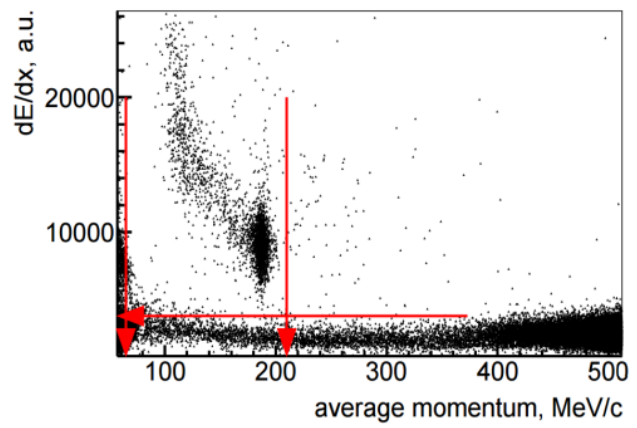

Fig. 3. The ionization losses vs momentum for positive tracks for data at $E_{c . m .}=1.06 \mathrm{GeV}$. Line shows selection of signal koans.

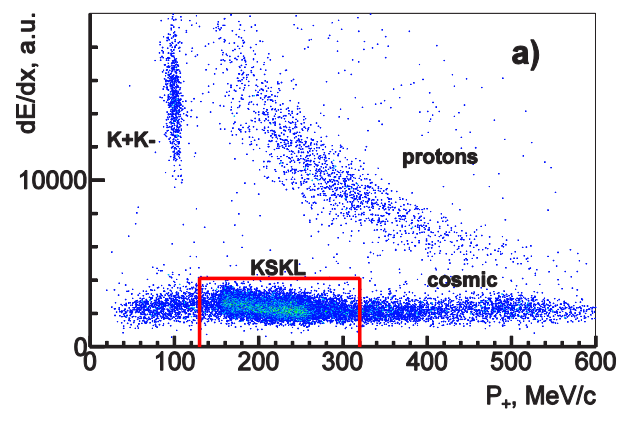

Fig. 4. The ionisation losses in DC vs momentum for positively charged pions for data at energy $E_{c . m .}=1.01 \mathrm{GeV}$. The line shows selections of pions from the $K_{S}$ decay.

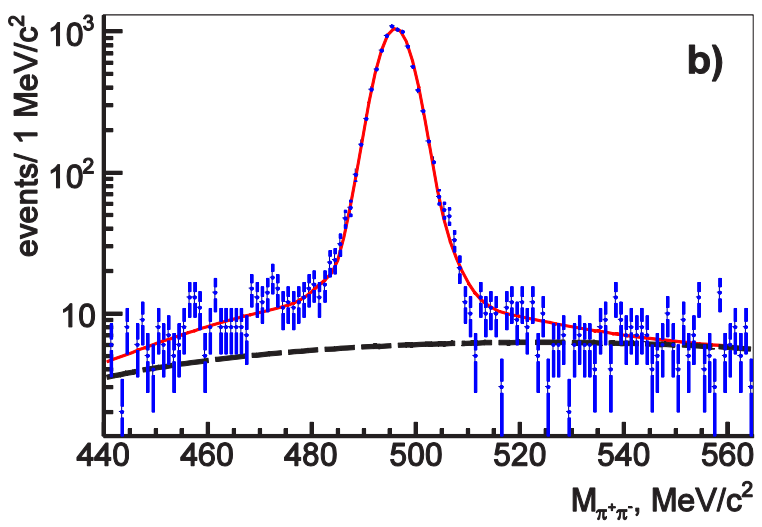

Fig. 5. Signal/background separation at $E_{\text {c.m. }}=1.01 \mathrm{GeV}$ by the approximation of the $m_{\text {inv }}\left(\pi^{+}, \pi^{-}\right)$distribution. Dashed line corresponds to the background, solid line - to the signal with background. 
The measured $e^{+} e^{-} \rightarrow K_{S} K_{L}, K^{+} K^{-}$cross sections together with their approximation are shown in Figs. 6-7 respectively. Systematic uncertainty is estimated to be $3 \%$ in charged channel and $1.8 \%$ in neutral channel. The final results for $K_{S} K_{L}$ are published in [8]. The cross sections were approximated according to the Vector Dominance Model:

$$
\sigma_{K K}=\frac{8 \pi \alpha}{3 s^{5 / 2}} p_{K}^{3}\left|\frac{g_{\phi \gamma} g_{\phi K K}}{D_{\phi}(s)} \pm A_{\rho}+A_{\omega}+A\right|^{2},
$$

where $D_{\phi}(s)$ is the denominator of the $\phi$ propagator, $A_{\rho / \omega}$ are the functions describing contributions of $\rho(770)$ and $\omega(782)$ resonances, constant $A$ describes the contribution of higher resonances. The sign «+» before the $A_{\rho}$ in formula (1) corresponds to $K^{+} K^{-}$ channel, «-»- to $K_{S} K_{L}$ channel. Further, the couplings $g_{\phi \gamma}$ and $g_{\phi K K}$ are equal

$$
\begin{array}{r}
g_{\phi \gamma}=\sqrt{\frac{3 m^{3}{ }_{\phi} \Gamma_{\phi} \mathcal{B}\left(\phi \rightarrow e^{+} e^{-}\right)}{4 \pi \alpha}}, \\
g_{\phi K K}=\sqrt{\frac{6 \pi m^{2}{ }_{\phi} \Gamma_{\phi} \mathcal{B}\left(\phi \rightarrow K^{+} K^{-}\right)}{p^{3}{ }_{K}\left(m_{\phi}\right)}} .
\end{array}
$$

The results of the approximation procedure in comparison with the PDG values (see [9]) are shown in Table 1. The contributions from the $\rho(770)$ and $\omega(782)$ were fixed from quark model and $\mathrm{SU}(3)$ symmetry of u-, d- and s-quarks. But if we introduce in the fit the additional floating parameter $r_{\rho, \omega}$, describing the relative deviation of the $g_{\rho K K}$ and $g_{\omega K K}$ from their SU(3)-predicted values, we obtain a compatible with unity result $r_{\rho, \omega}=0.76 \pm$ 0.11 . It is seen, that systematic errors dominate the uncertainty, and we still are working on the improvements.

\begin{tabular}{|c|c|c|}
\hline Parameter & CMD-3 & PDG \\
\hline$m_{\phi}, \mathrm{MeV}$ & $1019.468 \pm 0.005_{\text {stat }} \pm 0.06_{\text {syst }} \pm 0.02_{\text {model }}$ & $1019.461 \pm 0.019$ \\
\hline$\Gamma_{\phi}, \mathrm{MeV}$ & $4.254 \pm 0.009_{\text {stat }} \pm 0.016_{\text {syst }} \pm 0.012_{\text {model }}$ & $4.266 \pm 0.031$ \\
\hline $\begin{array}{c}\mathcal{B}\left(\phi \rightarrow e^{+} e^{-}\right) \times \\
\mathcal{B}\left(\phi \rightarrow K_{S} K_{L}\right) \times 10^{-5}\end{array}$ & $10.06 \pm 0.03_{\text {stat }} \pm 0.22_{\text {syst }} \pm 0.02_{\text {model }}$ & $10.10 \pm 0.13$ \\
\hline $\begin{array}{c}\mathcal{B}\left(\phi \rightarrow e^{+} e^{-}\right) \times \\
\mathcal{B}\left(\phi \rightarrow K^{+} K^{-}\right) \times 10^{-5}\end{array}$ & $15.83 \pm 0.04_{\text {stat }} \pm 0.47_{\text {syst }} \pm 0.1_{\text {model }}$ & $14.46 \pm 0.23$ \\
\hline $\begin{array}{c}\Gamma_{\phi \rightarrow e^{+} e^{-}-\mathcal{B}\left(\phi \rightarrow K^{+} K^{-}\right) \times} \\
10^{-1} \mathrm{keV}\end{array}$ & $6.735 \pm 0.014_{\text {stat }} \pm 0.199_{\text {syst }} \pm 0.042_{\text {model }}$ & $6.340 \pm 0.08$ \\
\hline
\end{tabular}

Table 1. The results of the approximation of the cross sections of $e^{+} e^{-} \rightarrow K_{S} K_{L}, K^{+} K^{-}$processes measured with the CMD-3 detector in comparison with PDG values.

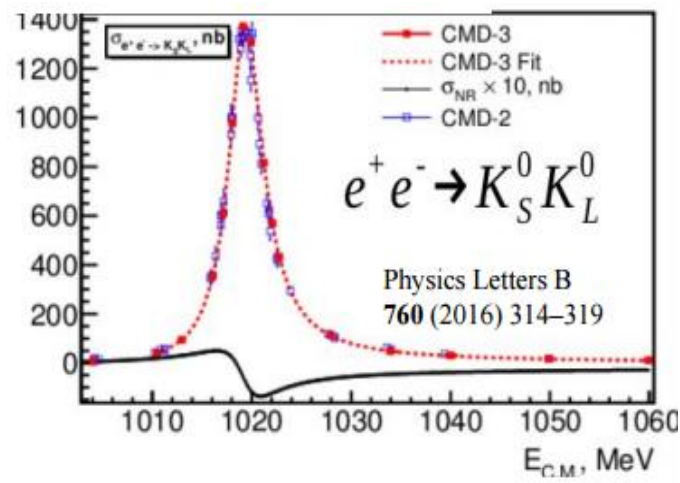

Fig. 6. Cross section of $e^{+} e^{-} \rightarrow K_{S} K_{L}$ process,

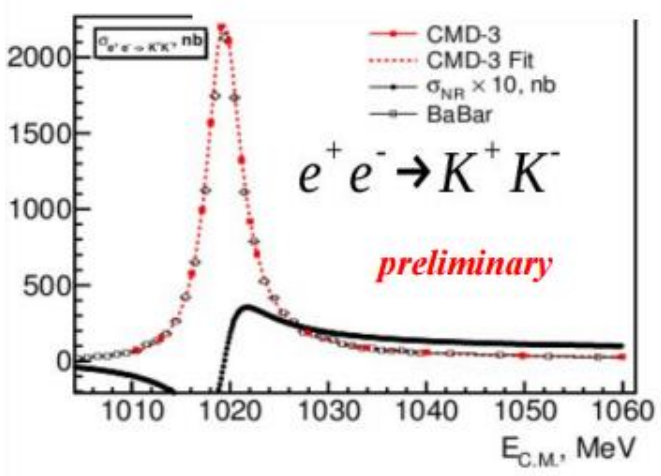

Fig. 7. Cross section of $e^{+} e^{-} \rightarrow K_{S} K_{L}$ process 
measured with the CMD-3 detector, together with its approximation. (preliminary results), measured with the CMD-3 detector, together with its approximation.

The combined analysis of $e^{+} e^{-} \rightarrow K_{S} K_{L}, K^{+} K^{-}$processes provides a possibility to check the precision of the isospin invariance of the $\mathrm{u}$ - and d-quarks, which predicts the value of unity to the factor

$$
R=\frac{g_{\phi K^{+} K^{-}}}{g_{\phi K_{S} K_{L}} \sqrt{Z\left(m_{\phi}\right)}}=\sqrt{\frac{\mathcal{B}\left(\phi \rightarrow K^{+} K^{-}\right) p^{3} K_{S}}{\mathcal{B}\left(\phi \rightarrow K_{S} K_{L}\right) p^{3}{ }^{+}} \frac{1}{Z\left(m_{\phi}\right)}},
$$

where $p_{K^{+}}$and $p_{K_{S}}$ are the momenta of charged and neutral kaons in $\phi \rightarrow K \bar{K}$ decays at $E_{c . m .}=m_{\phi}$, and

$$
Z\left(m_{\phi}\right)=\frac{\pi \alpha / \beta}{1-e^{-\pi \alpha / \beta}}\left(1+\frac{\alpha^{2}}{4 \beta^{2}}\right)
$$

is the Sommerfeld-Gamov-Sakharov factor, which takes into account the Coulomb interaction of charged kaons in the final state ( $\alpha$ is the fine structure constant, $\beta$ is the velocity of charged kaons over the speed of light). The previous measurements gave $R_{S N D}=0.92 \pm 0.03, R_{C M D-2}=0.943 \pm 0.013, R_{B a B a r}=0.972 \pm 0.017$ (see [9]) and one can suspect a significant deviation of $R$ from unity. Our measurement gives $R_{C M D-3}=$ $0.984 \pm 0.029$, and thus we do not see statistically significant deviation of $R$ from unity.

\section{Study of the $e^{+} e^{-} \rightarrow \pi^{+} \pi^{-} 2 \pi^{0}, 2 \pi^{+} 2 \pi^{-}$processes at $\phi$}

\subsection{Study of the $\mathrm{e}^{+} \mathrm{e}^{-} \rightarrow \pi^{+} \pi^{-} 2 \pi^{0}$ process}

To select the $\pi^{+} \pi^{-} 2 \pi^{0}$ final state events, we perform the $5 \mathrm{C}$ kinematic fit with all the combinations of 4 photons and 2 DC-tracks with opposite curvatures in the event, demanding the energy and momentum conservation, as well as the invariant mass of one pair of photons to be fixed at $\pi^{0}$ mass. Having selected the combination with the smallest $\chi^{2}{ }_{5 C}$, we apply the cut $\chi^{2}{ }_{5 C}<30$ to suppress the background (see Fig. 8). For the signal/background separation the approximation of the invariant mass of the second pair of photons (the invariant mass of which was not fixed at $m_{\pi^{0}}$ in the kinematic fit) is used (see Fig. 9). The signal and background shapes were taken from simulation with additional smearing.

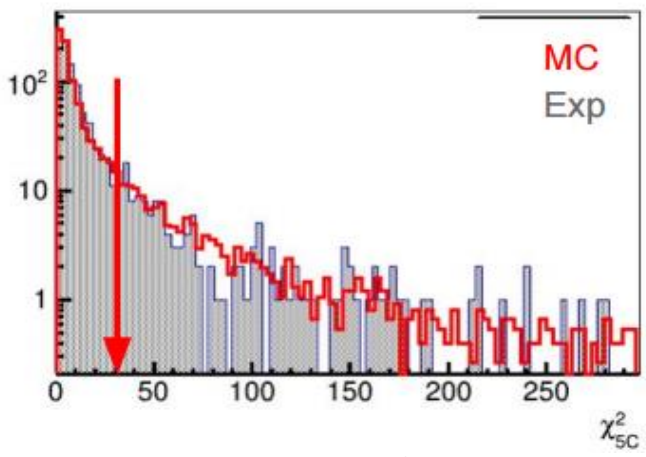

Fig. 8. The comparison of $\chi^{2}{ }_{5 C}$ distributions for data (filled histogram) and simulation (open histogram) at $E_{c . m .}=1.01 \mathrm{GeV}$.

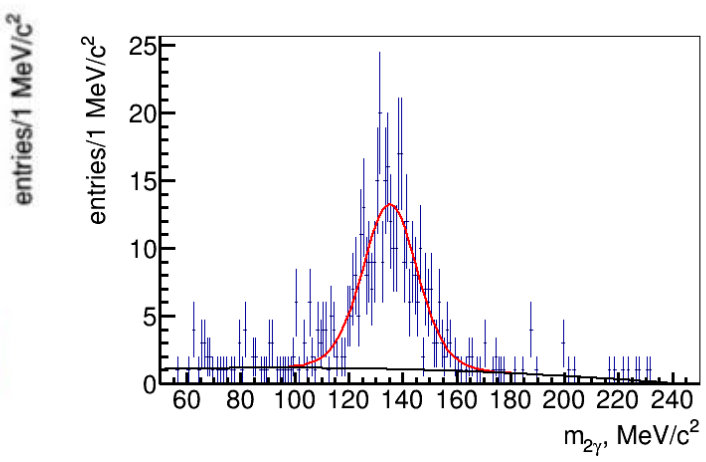

Fig. 9. The approximation of the invariant mass of the second pair of photons at $E_{c . m .}=1.01 \mathrm{GeV}$. 
The calculated $e^{+} e^{-} \rightarrow \pi^{+} \pi^{-} 2 \pi^{0}$ cross section together with its approximation is shown in Fig. 10. For the approximation we use the function

$$
\sigma_{\pi^{+} \pi^{-} 2 \pi^{0}}(\sqrt{s})=\sigma_{0} e^{A\left(\sqrt{s}-m_{\phi}\right)}\left|1-Z \frac{m_{\phi} \Gamma_{\phi}}{m^{2} \phi^{-s-i \sqrt{s} \Gamma_{\phi}}}\right|^{2},
$$

and the fit results are $\sigma_{0}=7.54 \pm 0.15 \mathrm{nb}, \operatorname{Re}(\mathrm{Z})=0.136 \pm 0.025, \operatorname{Im}(\mathrm{Z})=-0.084 \pm$ 0.023 . From these results one can obtain the value

$$
\mathcal{B}\left(\phi \rightarrow \pi^{+} \pi^{-} 2 \pi^{0}\right)=\frac{\sigma_{0}|Z|^{2} m^{2} \phi}{12 \pi \mathcal{B}\left(\phi \rightarrow e^{+} e^{-}\right)}=(5.3 \pm 1.6 \pm 0.8) \cdot 10^{-5},
$$

compatible with that in PDG [9]: $\mathcal{B}\left(\phi \rightarrow \pi^{+} \pi^{-} 2 \pi^{0}\right)=(4.7 \pm 0.5) \cdot 10^{-5}$.

Finally, it should be noted, that the major intermediate mechanisms of this process in the $\phi$ energy range are $e^{+} e^{-} \rightarrow \omega \pi^{0}$ and $e^{+} e^{-} \rightarrow a_{1} \pi$, and the determination of their amplitudes and relative phase via the partial wave analysis can be performed.

\subsection{Study of the $\mathrm{e}^{+} \mathrm{e}^{-} \rightarrow 2 \pi^{+} 2 \pi^{-}$process}

To extract the signal, we select the events with 3 or 4 DC-tracks, passing from the beams interaction region and having $d E / d x$, typical for pions. The major background processes were found to be $e^{+} e^{-} \rightarrow \phi \rightarrow \pi^{+} \pi^{-} \pi^{0}, K^{+} K^{-}, K_{S} K_{L}$ and $e^{+} e^{-} \rightarrow 2 e^{+} 2 e^{-}$. The $\pi^{+} \pi^{-} \pi^{0}$ final state is suppressed by the cut on the recoil masses of all the pairs of detected pions: $m_{\text {recoil }}\left(\pi_{i}, \pi_{j}\right)>2 m_{\pi}$. The $K^{+} K^{-}$contribution is suppressed by the cuts on $d E / d x$. The background from $K_{S} K_{L}$ final state is suppressed by the cuts on $K_{S}$ momentum and the invariant mass of two oppositely charged pions (see Fig. 11). Finally, Figs. 12-13 show the distributions of the energy disbalance in the events with four and three tracks, respectively. It is seen, that in the 4-track class we obtain almost background-free sample of signal events, whereas in 3-track class the signal/background separation is performed via the approximation of the energy disbalance distribution.

We determine the detection efficiency using the simulation of the signal process according to $e^{+} e^{-} \rightarrow a^{ \pm}{ }_{1} \pi^{\mp}$ model, as this intermediate mechanism is known to dominate the process in the region close to reaction threshold. From the Figs. 14a-b one can see good agreement between the polar angle distributions of charged pions in the experiment and simulation for 4-track and 3-track events, respectively.

The measured $e^{+} e^{-} \rightarrow 2 \pi^{+} 2 \pi^{-}$cross section together with its approximation using the formula (6) is shown in Fig. 15. The overall systematic uncertainty of cross section measurement is estimated to be $3.6 \%$. The fit results are: $\sigma_{0}=1.263 \pm 0.027 \mathrm{nb}, \operatorname{Re}(\mathrm{Z})=$ $0.146 \pm 0.030, \operatorname{Im}(Z)=-0.002 \pm 0.024$. From these one can obtain using the formula analogous to (7) the value

$$
\mathcal{B}\left(\phi \rightarrow 2 \pi^{+} 2 \pi^{-}\right)=(6.5 \pm 2.7 \pm 1.7) \cdot 10^{-6},
$$

which is in agreement with previous CMD-2 measurement $\mathcal{B}_{C M D-2}\left(\phi \rightarrow 2 \pi^{+} 2 \pi^{-}\right)=$ $\left(4.0_{-2.0}^{+2.8}\right) \cdot 10^{-6}[10]$, as well as with the estimation according to the vacuum polarization (which assumes $\operatorname{Im}(Z)=0$ ):

$$
\mathcal{B}\left(\phi \rightarrow \gamma^{*} \rightarrow 2 \pi^{+} 2 \pi^{-}\right)=\frac{9 \sigma_{0}\left(\mathcal{B}\left(\phi \rightarrow e^{+} e^{-}\right)\right)^{2} m^{2} \phi}{12 \pi \mathcal{B}\left(\phi \rightarrow e^{+} e^{-}\right)}=4.8 \cdot 10^{-6} .
$$




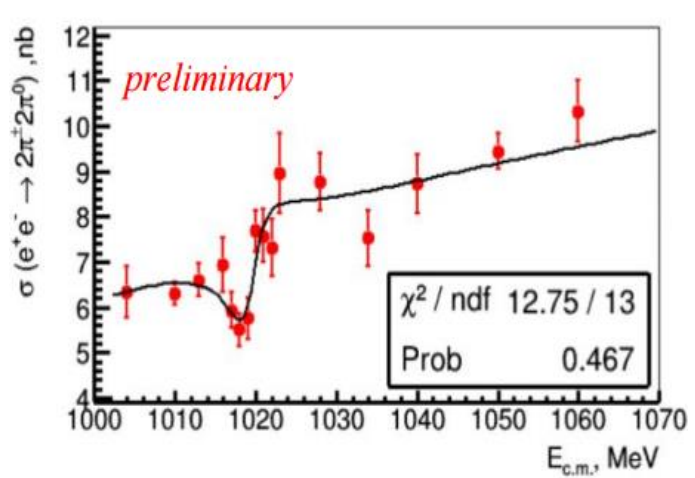

Fig. 10. Cross section of $e^{+} e^{-} \rightarrow \pi^{+} \pi^{-} 2 \pi^{0}$ process, measured with the CMD-3 detector, together with its approximation.

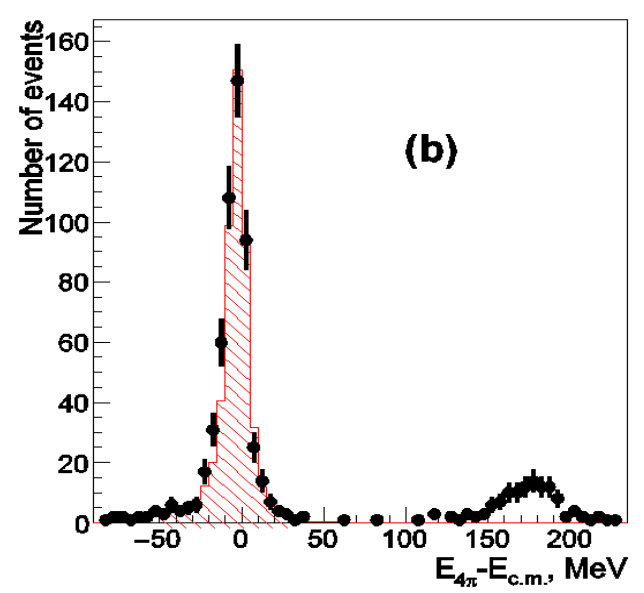

Fig. 12. The distribution of the energy disbalance for the events with four tracks at $E_{\text {c.m. }}=958 \mathrm{MeV}$.

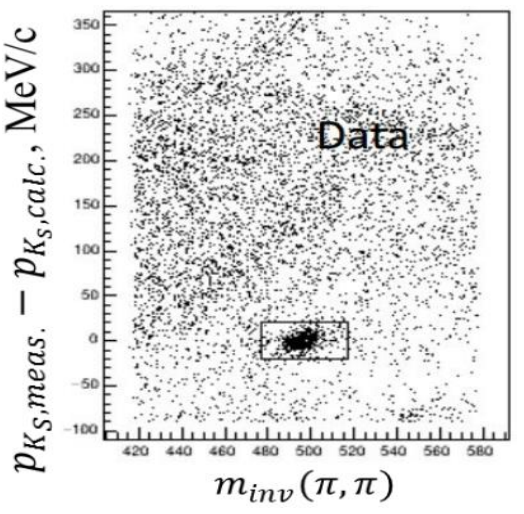

Fig. 11. The distribution of the

$\left(p_{K_{S, \text { measured }}}-p_{K_{L}, \text { calculated }}\right)$ versus the invariant mass of two oppositely charged pions in the experiment (data from all $E_{c . m}$. are used). The events in the frame (mainly $K_{S} K_{L}$ ) are rejected.

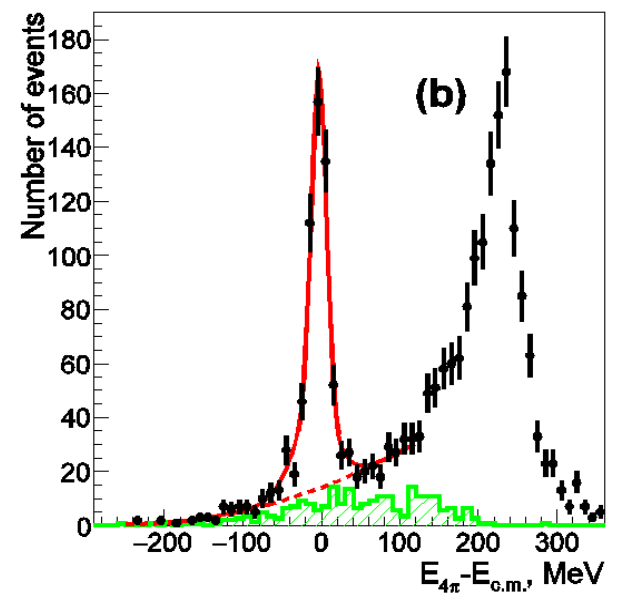

Fig. 13. The distribution of the energy disbalance for the events with three tracks at $E_{c . m .}=958 \mathrm{MeV}$. The dashed histogram represents the contribution of the $\pi^{+} \pi^{-} \pi^{0}$ final state. The peak at $\sim 250 \mathrm{MeV}$ is due to events of $e^{+} e^{-} \rightarrow 2 e^{+} 2 e^{-}$process. 

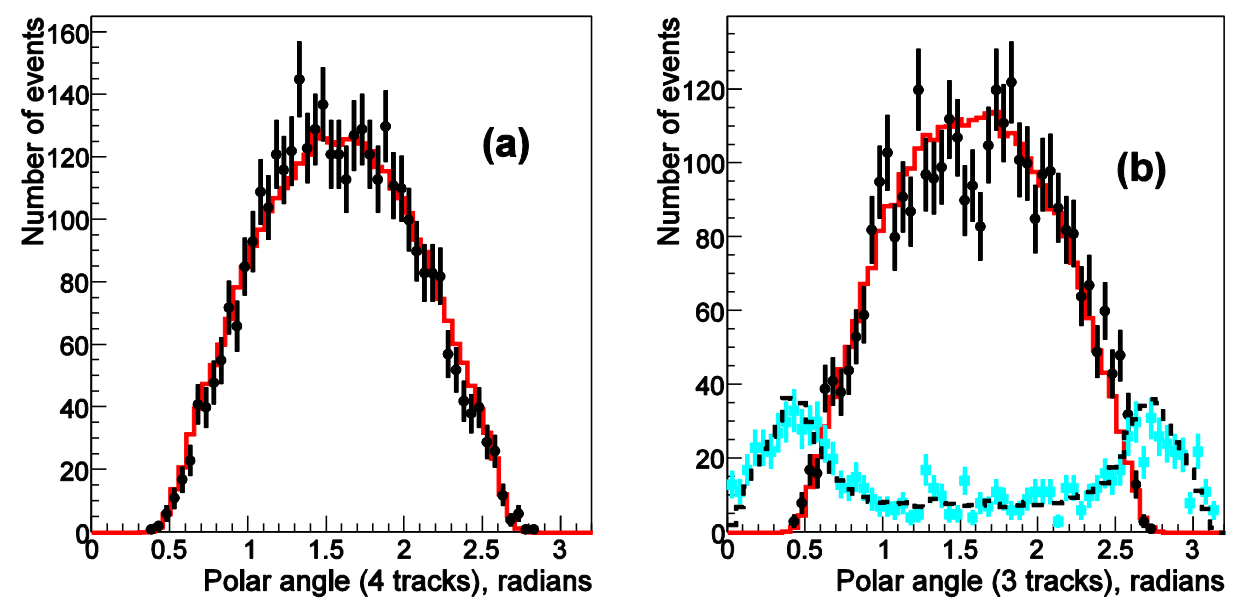

Fig. 14. The polar angle distributions of charged pions in the experiment (circle markers) and simulation (red open histogram) for 4-track (a) and 3-track (b) events, respectively (data from all $E_{c . m .}$ are used). For 3-track events also the distributions of the polar angle of missed pion in the experiment (square markers) and simulation (dashed line) are shown.

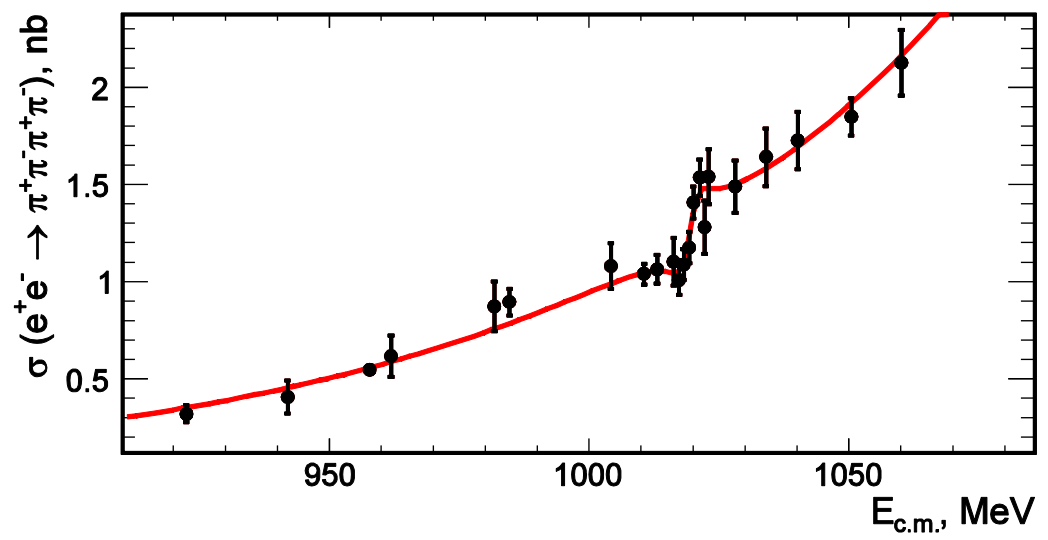

Fig. 15. Cross section of the $e^{+} e^{-} \rightarrow 2 \pi^{+} 2 \pi^{-}$process, measured with the CMD-3 detector, together with its approximation.

\section{Study of the $e^{+} e^{-} \rightarrow \eta \gamma$ process at $\phi$}

The $e^{+} e^{-} \rightarrow \eta \gamma$ process was studied in $\eta \rightarrow 2 \gamma$ decay mode in the $E_{c . m}$. range from 1.0 to $1.03 \mathrm{GeV}$ on the base of $5.5 \mathrm{pb}^{-1}$ of integrated luminosity. To select the signal, we consider the events, having at least 3 photons with the polar angles in the range from 0.42 to $\pi-0.42 \mathrm{rad}$. Then the kinematic cuts, as shown in Fig. 16, are applied. After that the major background processes are $e^{+} e^{-} \rightarrow \pi^{0} \gamma$ and $e^{+} e^{-} \rightarrow 3 \gamma$. We perform the kinematic fit, requiring the energy and momentum conservation and considering the common vertex of three photons as a floating parameter. After kinematic fit we arrange 3 photons according to their energies: $E_{\gamma_{1}}>E_{\gamma_{2}}>E_{\gamma_{3}}$ (see $m_{\text {inv }}\left(\gamma_{1}, \gamma_{3}\right)$ vs. $m_{i n v}\left(\gamma_{2}, \gamma_{3}\right)$ distribution in Fig, 17). As it follows from the kinematics, in the $\phi$-meson energy region the monochromatic photon in $\eta \gamma \rightarrow 3 \gamma$ reaction has, in the majority of cases, the medium energy $E_{\gamma_{2}}$. 
Signal/background separation is performed using the approximation of the $m_{i n v}\left(\gamma_{1}, \gamma_{3}\right)$ distribution in the experiment (see Fig. 18). The preliminary results for the $e^{+} e^{-} \rightarrow \eta \gamma$ cross section measurement at $\phi$ are shown in Fig. 19. The systematic uncertainties are now being studied, and the major uncertainty is the difference between the photon detection efficiency in the Monte Carlo and experiment.

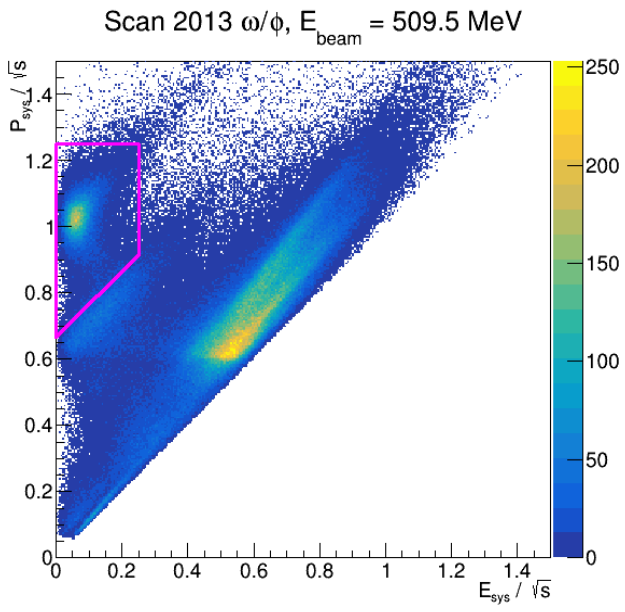

Fig. 16. The total energy vs. total momentum of all the combinations of three photons in the event (data from all $E_{c . m .}$ are used). Combination inside the frame is accepted.

Experimental data, $\mathrm{L}=581.2 \mathrm{nb}^{-1}$

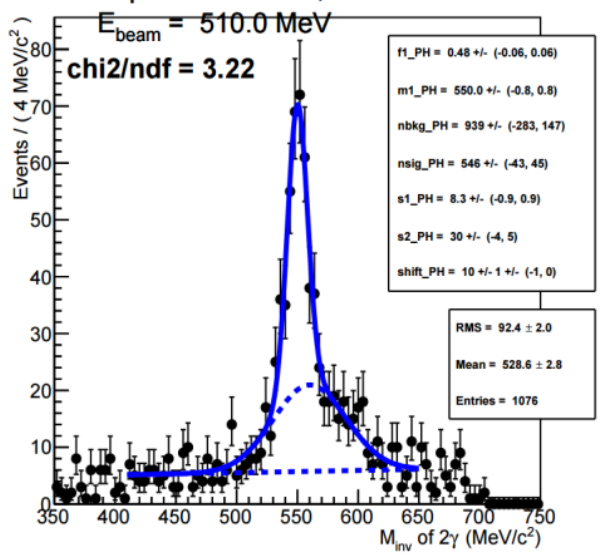

Fig. 18. Signal/background separation by the approximation of the $m_{i n v}\left(\gamma_{1}, \gamma_{3}\right)$ distribution in the experiment $\left(E_{\text {c.m. }}=1.02\right.$ $\mathrm{GeV}$ ).

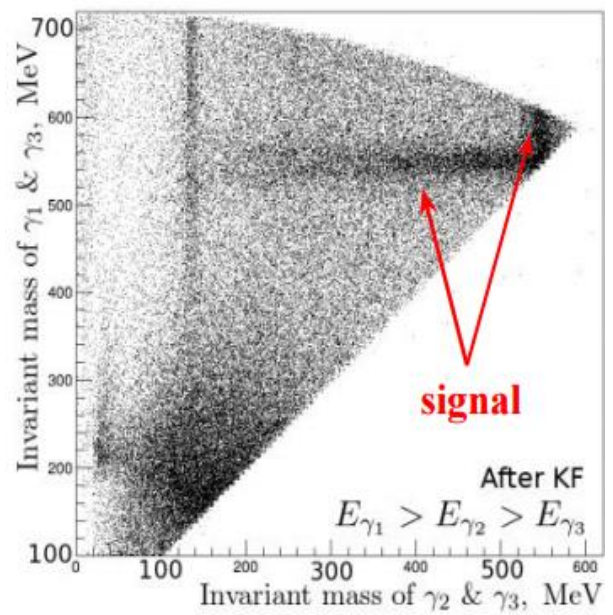

Fig. 17. The $m_{i n v}\left(\gamma_{1}, \gamma_{3}\right)$ vs. $m_{i n v}\left(\gamma_{2}, \gamma_{3}\right)$ distribution for the photons after kinematic fit (data from all $E_{c . m .}$ are used).

This study

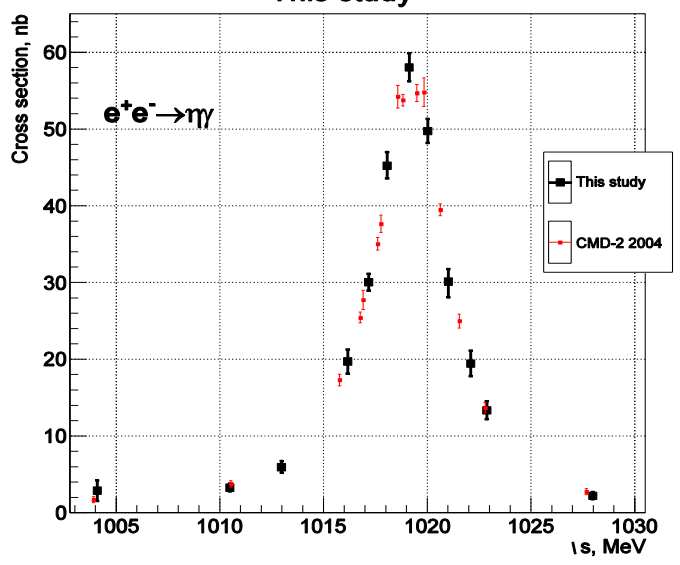

Fig. 19. The preliminary results for the $e^{+} e^{-} \rightarrow \eta \gamma$ cross section measurement in the $\phi$ meson region with the CMD-3 detector.

\section{Conclusion}

The recent results of the study of the processes $e^{+} e^{-} \rightarrow K_{S} K_{L}, K^{+} K^{-}, \pi^{+} \pi^{-} 2 \pi^{0}$, $2 \pi^{+} 2 \pi^{-}, \eta \gamma$ at the $\phi$-meson region with the CMD-3 detector at the VEPP-2000 collider were described. From the combined analysis of $K_{S} K_{L}$ and $K^{+} K^{-}$final states the $\phi$-meson parameters were measured, and the obtained ratio of $g_{\phi K^{+} K^{-}}$and $g_{\phi K_{S} K_{L}}$ couplings was found to be compatible with the isospin symmetry. From the analyzes of the $\pi^{+} \pi^{-} 2 \pi^{0}$ and 
$2 \pi^{+} 2 \pi^{-}$final states the branching ratios $\mathcal{B}\left(\phi \rightarrow \pi^{+} \pi^{-} 2 \pi^{0}\right)$ and $\mathcal{B}\left(\phi \rightarrow 2 \pi^{+} 2 \pi^{-}\right)$were determined. We still are working on the reduction of the systematic uncertainties in the study of processes $e^{+} e^{-} \rightarrow K^{+} K^{-}, \pi^{+} \pi^{-} 2 \pi^{0}, \eta \gamma$.

We thank the VEPP-2000 personnel for the excellent machine operation. Part of this work is supported by the Russian Foundation for Basic Research grants RFBR 15-02-05674-a and RFBR 1602-00160-a.

\section{References}

1. K. Hagiwara, R. Liao, A.D. Martin, D. Nomura, T. Teubner, J. Phys. G 38, 085003 (2011)

2. G.W. Bennett et al. (Muon g-2 Collaboration), Phys. Rev. D 73, 072003, (2006)

3. D.E. Berkaev et al., JETP 113, 213 (2011)

4. B.I. Khazin et al. (CMD-3 collaboration), Nucl. Phys. B (Proc. Suppl.) 181-182, 376 (2008)

5. V.M. Aulchenko et al., JINST 10, P10006 (2015)

6. R.R. Akhmetshin et al. (CMD-3 collaboration), Nucl. Phys. B (Proc. Suppl.) 225-227, 69 (2012)

7. E.V. Abakumova et al., Phys. Rev. Lett. 110, 140402 (2013)

8. E.A. Kozyrev et al., Phys. Lett. B 760, 314-319 (2016)

9. K.A. Olive et al., Particle Data Group, Chin. Phys. C 38, 090001, (2014)

10. R. R. Akhmetshin et al. (CMD-2 Collaboration), Phys. Lett. B 491, 81 (2000) 